\title{
Measurements in an incompressible three-dimensional turbulent boundary layer, under infinite swept-wing conditions, and comparison with theory
}

\author{
By B. VAN DEN BERG, A. ELSENAAR, \\ J. P. F. LINDHOUT \\ National Aerospace Laboratory, Amsterdam
}

AND P. WESSELING

Twente University of Technology, Enschede, The Netherlands

(Received 7 June 1974 and in revised form 4 December 1974)

First a three-dimensional turbulent boundary-layer experiment is described. This has been carried out with the specific aim of providing a test-case for calculation methods. Much attention has been paid to the design of the test set-up. An infinite swept-wing flow has been simulated with good accuracy. The initially twodimensional boundary layer on the test plate was subjected to an adverse pressure gradient, which led to three-dimensional separation near the trailing edge of the plate. Next, a calculation method for three-dimensional turbulent boundary layers is discussed. This solves the boundary-layer equations numerically by finite differences. The turbulent shear stress is obtained from a generalized version of Bradshaw's two-dimensional turbulent shear stress equation. The results of the calculations are compared with those of the experiment. Agreement is good over a considerable distance; but large discrepancies are apparent near the separation line.

\section{Introduction}

Up to now, most effort in turbulent boundary-layer research has been put into investigating two-dimensional boundary layers. A large number of calculation methods for two-dimensional turbulent boundary layers are available at present, and many measurements in all kinds of two-dimensional boundary layers have been carried out. Much less attention has been paid to threedimensional boundary layers, although nearly all boundary layers are threedimensional in practice. Over the last few years, however, the effort spent on the investigation of three-dimensional turbulent boundary layers has much increased. At this moment there exist a number of methods of calculation for three-dimensional turbulent boundary layers (Nash 1969; Bradshaw 1971; Pierce \& Klinksiek 1971; Michel, Cousteix \& Quemard 1972; Smith 1972; Krause 1973; Shanebrook \& Sumner 1973; Fannelop \& Humphreys 1974). Two of the present authors have developed such a method (Wesseling \& Lindhout 1971). This is based on an extension to three dimensions of the 
two-dimensional turbulent shear stress equation of Bradshaw, Ferriss \& Atwell (1967). The partial differential equations obtained are solved numerically by a finite-difference method. The method is general, being applicable to all boundary layers on surfaces of small curvature.

All turbulent boundary-layer calculation methods contain empirical information. Therefore it is essential to compare them with results of experiments. Although a number of experiments on three-dimensional boundary layers have been performed (e.g. Johnston 1960, 1970; Hornung \& Joubert 1963; Francis \& Pierce 1967; Bradshaw \& Terrell 1969; East and Hoxey $1969 a, b$; Cumpsty \& Head 1970; Vermeulen 1971; East 1973), nearly all of them are rather unsuited to testing the corresponding methods of calculation. Very often, insufficient data were obtained to define completely the flow conditions, such as the initial conditions of the boundary layer and the accurate surface static pressure distribution. Moreover, in several experiments three-dimensional boundary layers were created very abruptly, so that shear stresses played only a minor part in flow development. In the experiment to be described we attempted to avoid these shortcomings.

The model that was used for the measurements was designed with particular care. The boundary layer on the test surface of this model develops gradually from a two-dimensional, zero pressure gradient layer to a three-dimensional separation. For reasons of simplicity, both mathematical and experimental, it is attractive to simulate a quasi two-dimensional flow (i.e. flow conditions such as occur on an infinite swept wing). To obtain a sufficiently close approximation to the infinite swept-wing condition on the model, special measures had to be taken. The complicated design of the model will be discussed in more detail later.

The model was installed in the NLR 3 by $2 \mathrm{~m}$ low speed tunnel. Extensive measurements in the boundary layer were made. The magnitude and direction of the velocity were measured with a rotatable hot wire, up to very close to the surface. Wall Pitots of small height were employed for the determination of the wall shear stress. (Turbulence measurements are now being carried out; a detailed description of these will be published in the future.)

\section{Experiment}

\subsection{Model design}

To obtain a test set-up suitable for checking three-dimensional boundary-layer calculation methods, the following requirements were made of the model to be used.

(i) A flow similar to that which occurs on an infinite swept wing should be simulated. From a physical point of view, the boundary-layer flow on an infinite swept wing is not different from a general, three-dimensional, boundary layer. Mathematically, however, a quasi two-dimensional flow is much easier to treat. By measuring a boundary layer under infinite swept-wing conditions, it is also possible to involve calculation methods that are restricted to the infinite sweptwing case in a comparison of theory and experiment. Apart from this, there is the additional advantage that, under these conditions, it is sufficient to carry out 


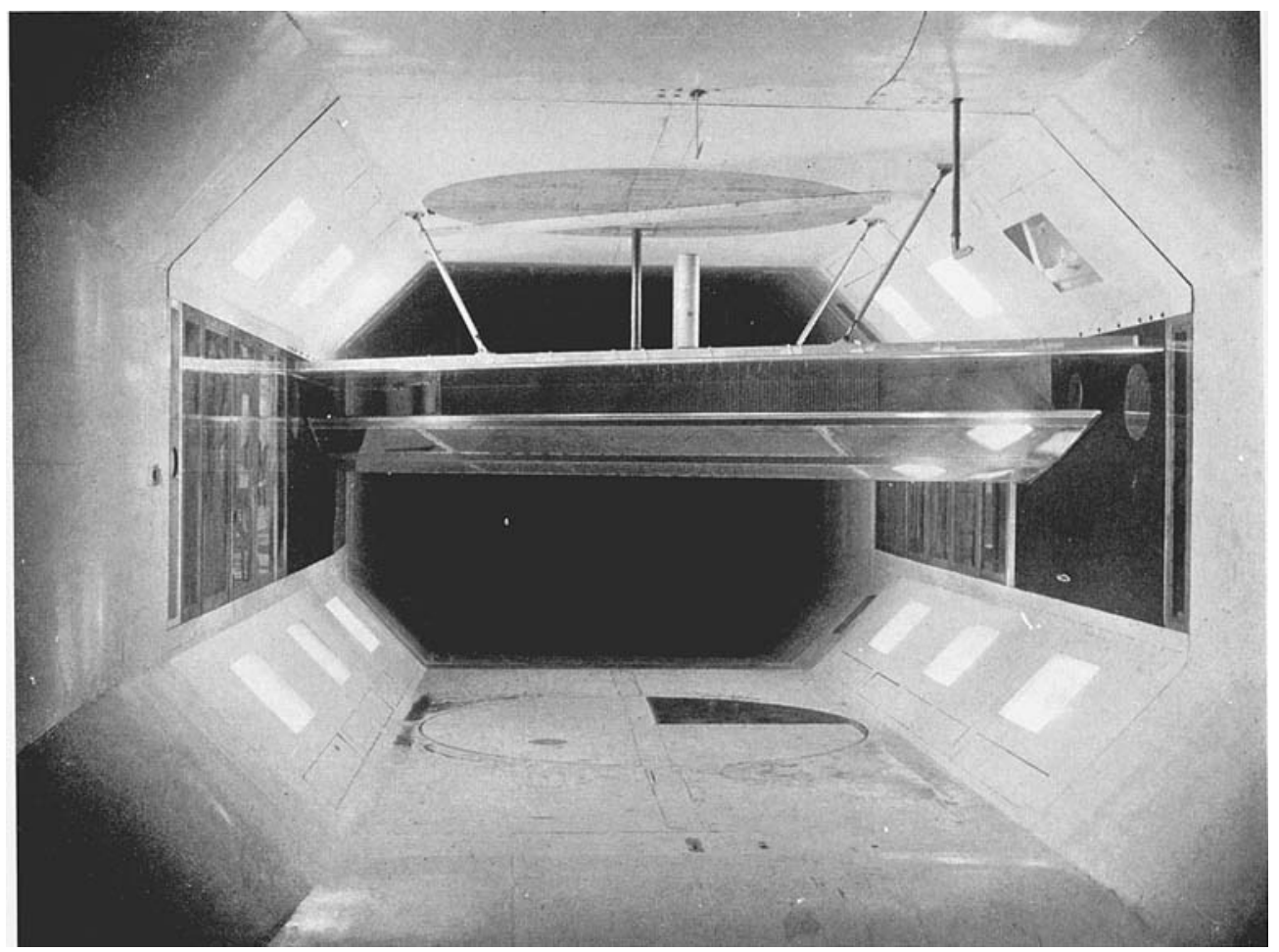

Figure 1. Photograph of the model in the wind tunnel.

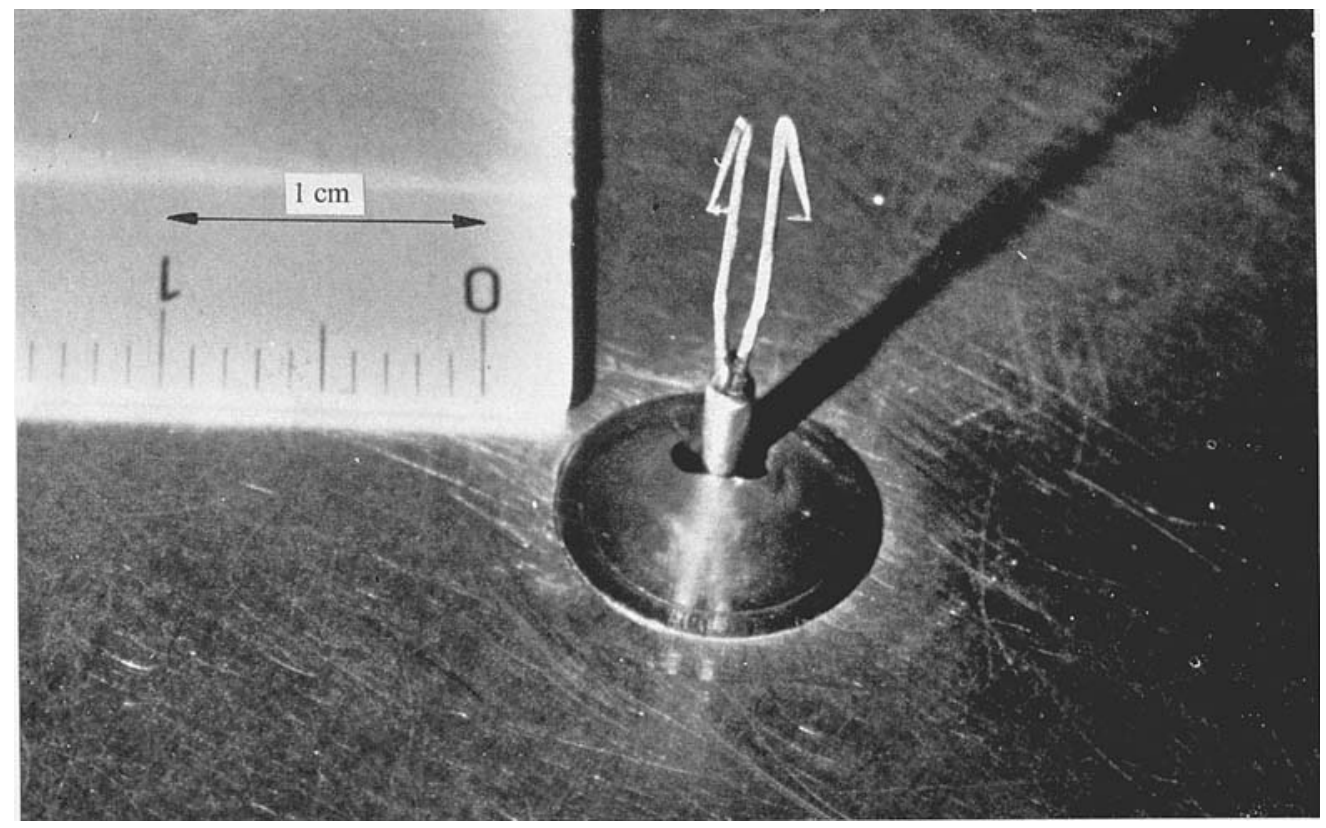

Figure 3. View of rotatable hot-wire probe. 



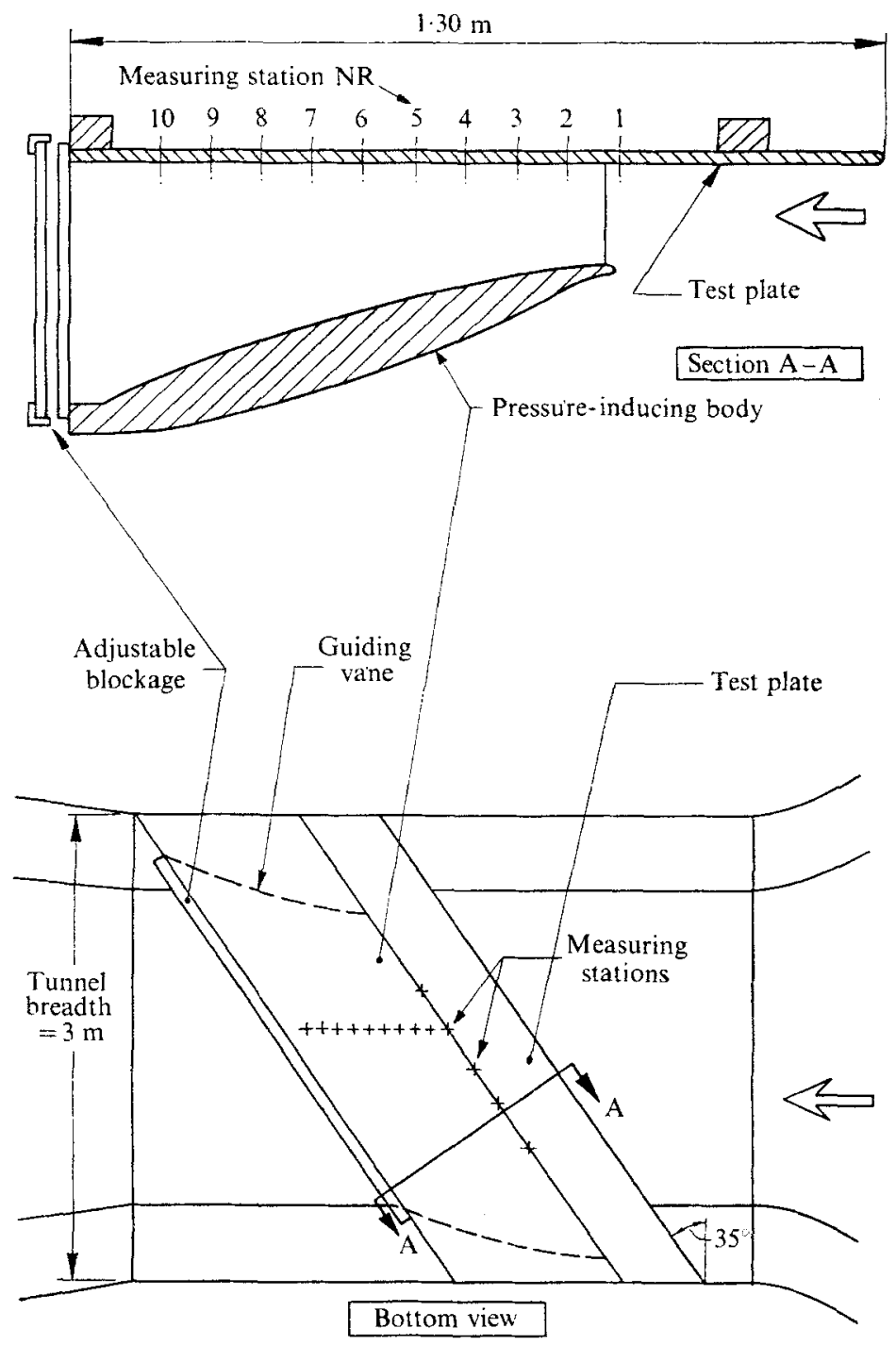

Figure 2. Sketch of the model.

measurements at one section only. This leads to a considerable reduction in the amount of experimental data required.

(ii) The pressure should be constant along the most forward part of the model. In that case, a normal two-dimensional constant-pressure boundary layer will develop there. The important advantage is that the initial conditions of the threedimensional boundary layer are then well known. After the constant-pressure region, the pressure should increase gradually in such a manner that a threedimensional separation occurs near the model trailing edge. This pressure rise should not be too steep. This requirement has not always been met by some earlier three-dimensional experiments, where boundary-layer separation took place after a distance much shorter than that in most practical cases. This con- 
siderably reduces the value of such an experiment as a check on semi-empirical calculation methods. Furthermore, in regions of rapid pressure variation, shear stresses play a minor role, but precisely the semi-empirical assumptions about the turbulent shear stress need experimental verification.

(iii) The measurements should be carried out in a boundary layer on a flat surface, to eliminate the as yet relatively unknown effects of surface curvature on the structure of turbulence. These effects are large according to present-day views (see e.g. Bradshaw 1973). Even the surface curvatures that occur on ordinary airfoils seem sufficient to affect boundary-layer development noticeably. A flat test surface also has experimental advantages.

On the basis of these requirements a model was designed consisting of a smooth flat plate with a pressure distribution induced on it by a large airfoil-like body, mounted near the plate. Both the flat test plate and the pressure-inducing body had an angle of sweep of $35^{\circ}$. Figure 1 (plate 1) shows a photograph of the model in the wind tunnel. A sketch of the model is given in figure 2. As appears from the sketch, the flow between the test plate and the pressure-inducing body may be regarded as a diverging channel flow. A diverging channel flow is required if the pressure is to increase in the direction of flow. The flow in front of the diverging channel should be parallel, to obtain a constant-pressure region along the most forward part of the flat plate (one of the requirements). A parallel flow can be achieved by introducing a blockage in the channel, of such a magnitude that the right amount of air is going through the channel. Such a flow blockage was obtained here by installing a large number of bars at the outlet of the channel. The magnitude of the blockage at the model trailing edge was adjustable, so that an initial constant-pressure region along the test plate could be approximated with good accuracy after some preliminary tests.

Another noteworthy feature of the model is the presence of guiding vanes, mounted between the pressure-inducing body and the test plate (see figure 2). Without these guiding vanes, it proved impossible to obtain a reasonably close approximation to the infinite swept-wing condition. The cause is the large effect of the constraint of the end walls on the flow in the case of sweep. To diminish the influence of the end walls, their shape should be adapted to the shape of the streamlines, according to potential flow theory for the infinite swept wing. For a channel flow (which here occurs approximately), this leads to simply curved end walls. Instead of adapting the shape of the tunnel walls, curved guiding vanes of the required shape were installed near the tunnel walls. Actually, the shape of the guiding vanes was determined experimentally by trial and error, in such a way that the pressure distribution on the test plate approximated the infinite swept-wing condition as accurately as possible.

To determine the pressure distribution on the test plate, the static pressure was measured at about 200 holes in the surface. Such a large number of static pressure holes is required if the pressure gradients are to be deduced with sufficient accuracy. Boundary-layer measurements were carried out at a number of stations, whose positions are indicated in figure 2. 


\subsection{Experimental techniques}

The direction and the magnitude of the velocity at the boundary-layer edge were measured with a cylindrical yaw tube and a Pitot static tube, respectively. The velocity measurements inside the boundary layer were carried out with a hot wire, which could be rotated. A photograph of the rotatable hot-wire probe, which resembles the probe described by Rogers \& Head (1969,) is given in figure 3 (plate 1). The photograph shows the probe protruding through the plate surface at one of the measuring stations. At the reverse side of the plate, a traversing mechanism was mounted, by which the probe could be translated and rotated. The measurements extended from outside the boundary layer up to a distance of $0.15 \mathrm{~mm}$ from the surface. The direction of the velocity was found by rotating the hot wire and subsequently determining the line of symmetry of the data. The hot wire was calibrated at the boundary-layer edge, where the magnitude of the velocity is known from Pitot static tube measurements. Calibrations were made before and after each traverse through the boundary layer. The traverse was repeated when the difference between both calibrations was larger than $1 \%$ in the velocity.

The magnitude of the wall shear stress was determined by wall Pitots of the Stanton type, with slit heights of $0.1 \mathrm{~mm}$ and $0.2 \mathrm{~mm}$. Both wall Pitots were calibrated in a long pipe with fully developed flow. The wall Pitots were rotated to establish the symmetry line, and thus the approximate direction of the wall shear stress. More details concerning the experimental techniques employed are given in van den Berg \& Elsenaar (1972).

\subsection{Results}

The measured pressure distribution on the flat plate is plotted isometrically in figure 4. On infinite swept wings, the pressure is constant along lines parallel to the leading edge. It is evident from figure 4 that generally the pressure variations parallel to the leading edge are relatively small. Near the trailing edge, there is, at the left, a region with larger spanwise pressure gradients. Since this region is situated far downstream and at some distance from the measurement plane, the effect on the flow in the measurement plane may be expected to be small, however. The degree of approximation to the infinite swept-wing condition will be investigated further in $\$ 4$, by comparing the results of flow calculations using the measured three-dimensional pressure distribution with those using an infinite swept-wing pressure distribution based on data in the measurement plane.

It is also evident from figure 4 that the pressure is nearly constant over a substantial length upstream of measuring station 1. This means that the boundary layer at this station will be nearly two-dimensional, in accordance with the requirement made.

Some results of velocity measurements in the boundary layer, with the rotatable hot-wire probe, are given in figures 5 and 6 . In figure 5 , the magnitude of the velocity at stations $1,4,7$ and 10 (see figure 2) is plotted against distance from the wall. The shape of the velocity profiles is seen to be quite normal, with the 


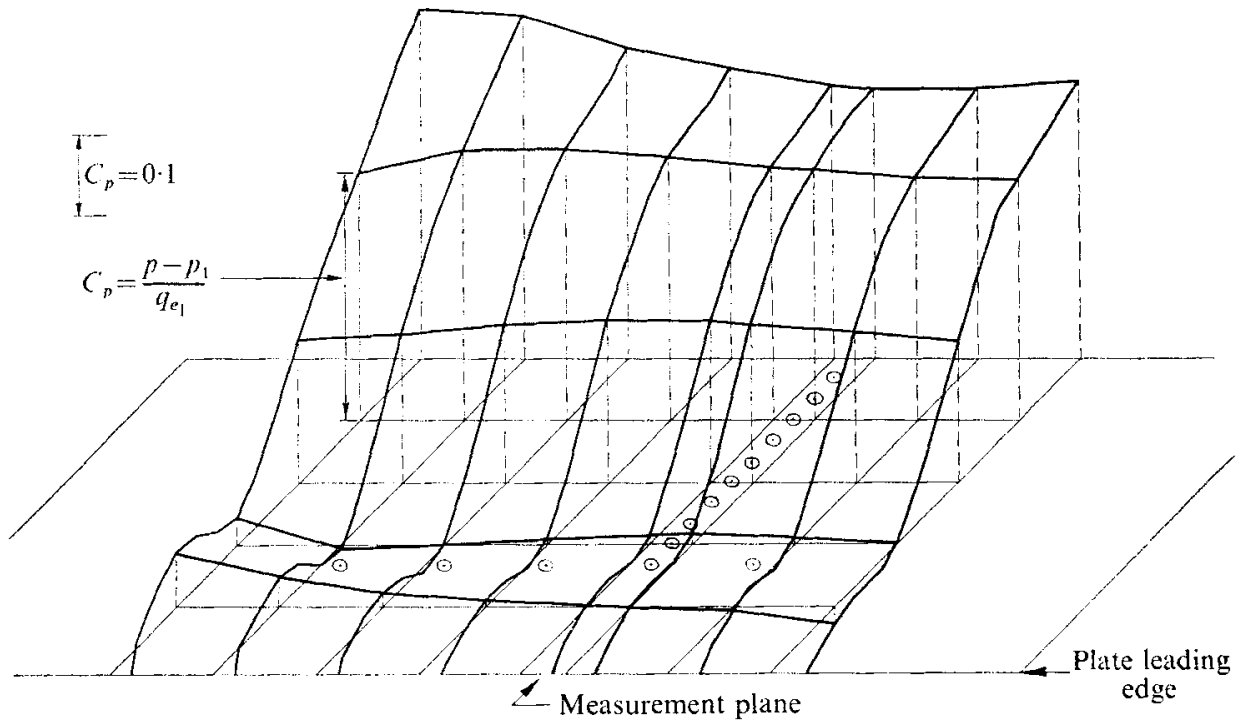

Frgure 4. Isometric plot of pressure distribution on the flat plate.

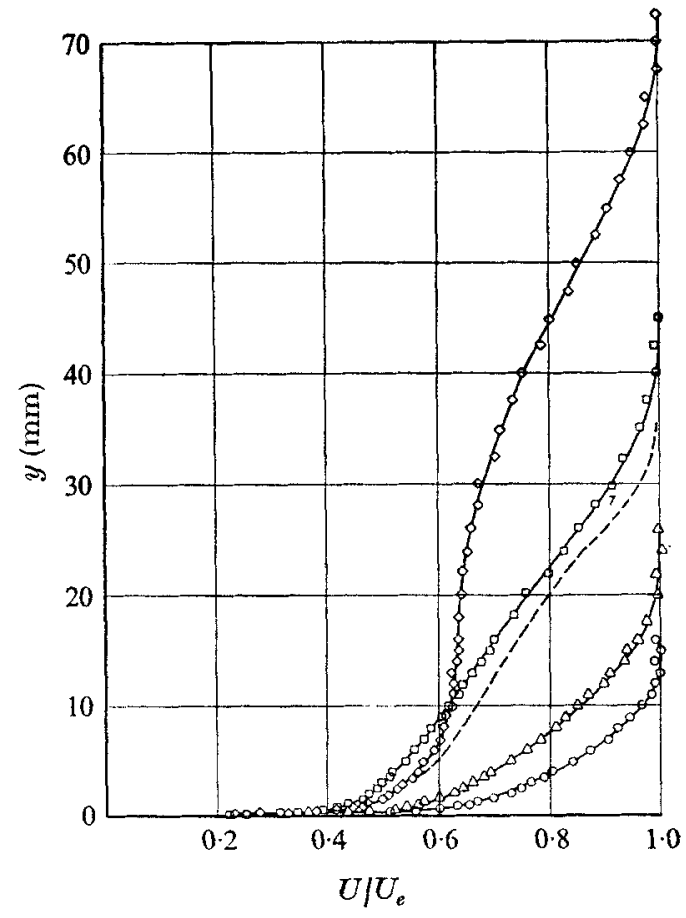

FIGURE 5

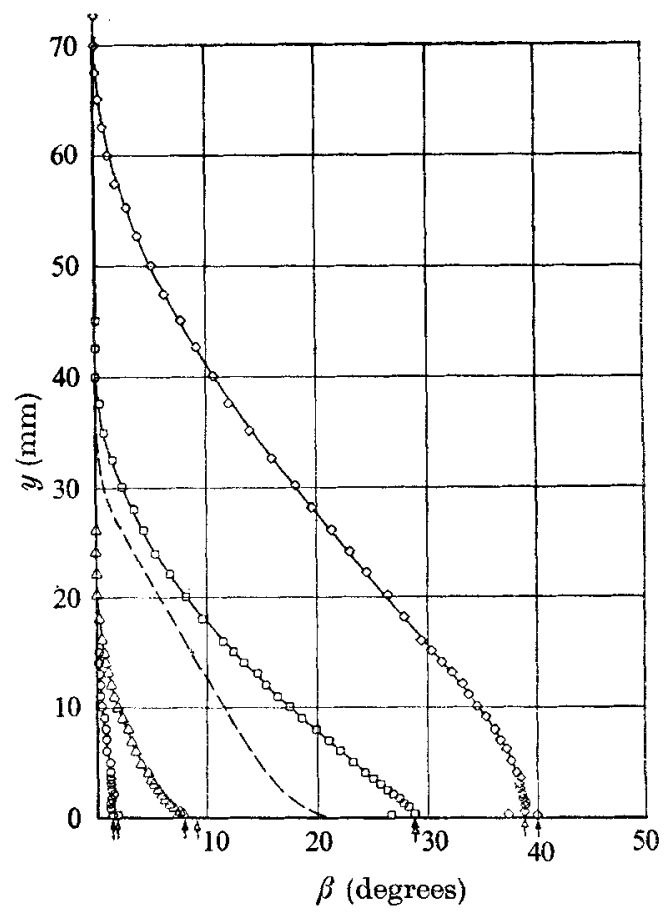

FiguRe 6

Figure 5. Absolute velocities in the boundary layer. - - - results of calculation; - measurements.
Station
$1 \quad 4$
$7 \quad 10$
$\square \quad \diamond$

FIGURE 6. Flow angles in the boundary layer. $\beta_{w}$ measured with 0.1 (个) and $0 \cdot 2(\uparrow) \mathrm{mm}$ wall Pitot. Symbol key otherwise as for figure 5 . 
exception perhaps of the velocity profile at station 10 , which exhibits a region with an approximately constant velocity in the middle of the boundary layer. This may be connected with the fact that station 10 is located behind the threedimensional separation line, as will be seen later. The shape of the initial velocity profile (station 1) corresponds well to the shape to be expected for a twodimensional zero pressure gradient boundary layer at the Reynolds number $U_{e} \theta / \nu=3290$ at this station.

In figure 6 , the measured flow angles in the boundary layer have been plotted. Large variations in flow direction are seen to occur, particularly at the downstream stations. In the graph the wall shear stress angles, established with wall Pitots, are also indicated. The trend of the angles found with the rotatable hot wire is seen to match well with the wall Pitot angles. Close to the wall, the scatter of the hot-wire data increases strikingly. This must be attributed to the very large velocity gradients there, and the consequent large errors in the angle found owing to a small height variation during rotation of the hot wire. It is evident from figure 6 that the flow at station 1 is not completely two-dimensional, but the flow angle variation is not more than $1.5^{\circ}$.

Measurements have also been carried out at stations located the same distance from the plate leading edge as station 1, but at different spanwise positions (see figure 2). It appeared from the data at these stations that the velocity profiles at the different spanwise positions coincide within the experimental scatter. This is a necessary condition when the flow on an infinite swept wing is to be simulated.

Figure 7 gives some typical Clauser plots (see Clauser 1954). The velocity is plotted there against the logarithm of the wall distance; consequently the region near the wall is shown very clearly. This kind of plot is used in two-dimensional flow, to deduce the wall shear stress. If it is assumed that the simple law of the wall

$$
U / u_{\tau}=(1 / k)\left[\ln \left(y u_{\tau} / \nu\right)+A\right]
$$

holds, a family of straight lines can be drawn in the graph with the wall shear stress $\tau_{w}$ or the skin-friction velocity $u_{\tau}=\left(\tau_{w} / \rho\right)^{\frac{1}{2}}$ as a parameter. It is evident from figure 7 that the measured velocity profiles coincide with a member of this family over a distance amply large enough to connect a wall shear stress value to the measured velocity profile. This result suggests that the two-dimensional law of the wall describes the variation of the velocity in this three-dimensional boundary layer with good accuracy, up to a fairly large distance from the wall. The absolute value of the velocity is plotted here. One could also have plotted the component of the velocity in the wall shear stress direction; but the difference is less than $0.5 \%$ in the region of interest.

In figure 8, the skin-friction coefficients $C_{f}=\tau_{w} / \frac{1}{2} \rho U_{e}^{2}$ deduced from the Clauser plots are compared with the values obtained with both wall Pitots. The height of these wall Pitots is so small that they are largely situated in the viscous sublayer. It is clear from figure 8 that the agreement between the skin-friction coefficients obtained in different ways is good, considering the accuracy of wall shear stress measurements. A fundamental doubt as to the methods used to determine the wall shear stress may be entertained, however, since in all cases one relies on the assumption that the two-dimensional law of the wall still holds in this three- 


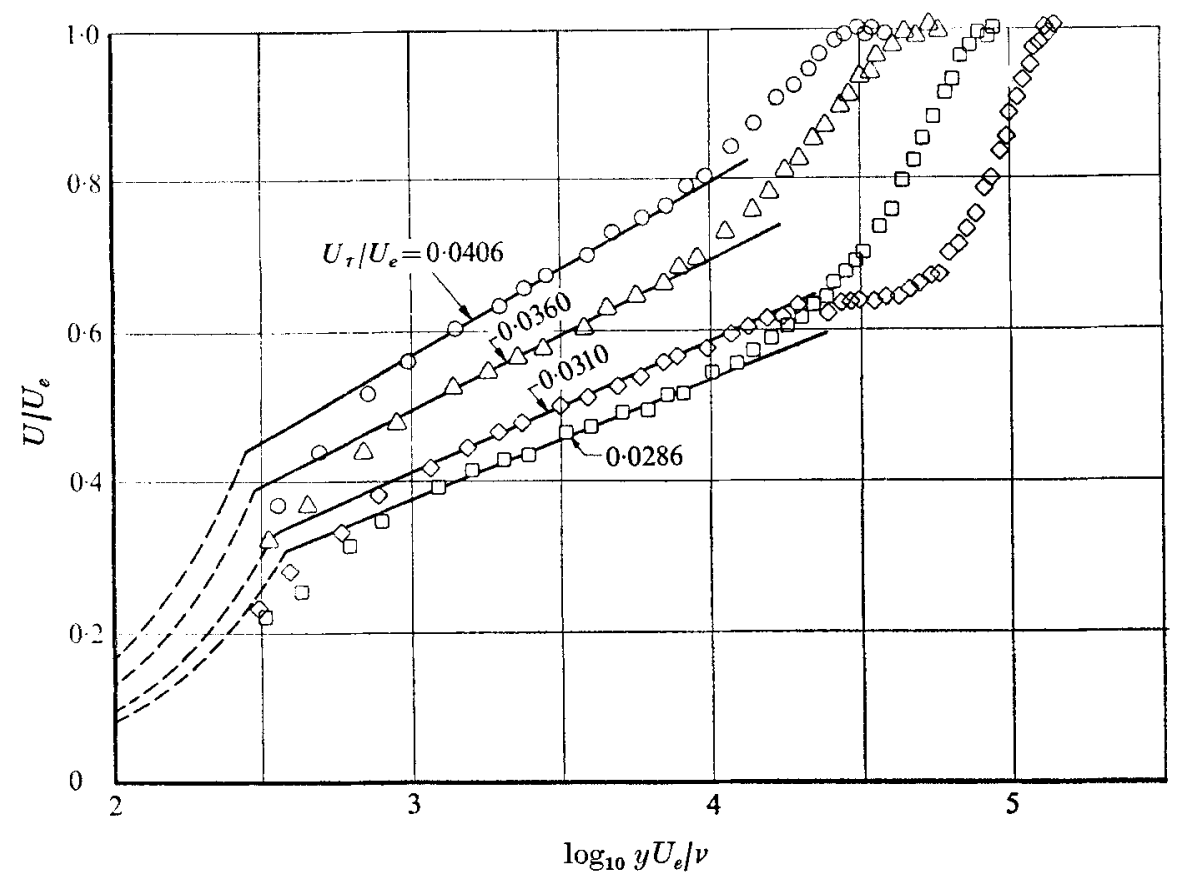

FIGURE 7. Clauser plot of absolute velocity. ---, $U / u_{\tau}=y u_{\tau} / \nu ;-, \log$ law, $U / u_{\tau}=1 / 0 \cdot 41\left[\ln \left(y u_{\tau} / \nu\right)+2 \cdot 05\right]$. Symbol key otherwise as for figure 5 .

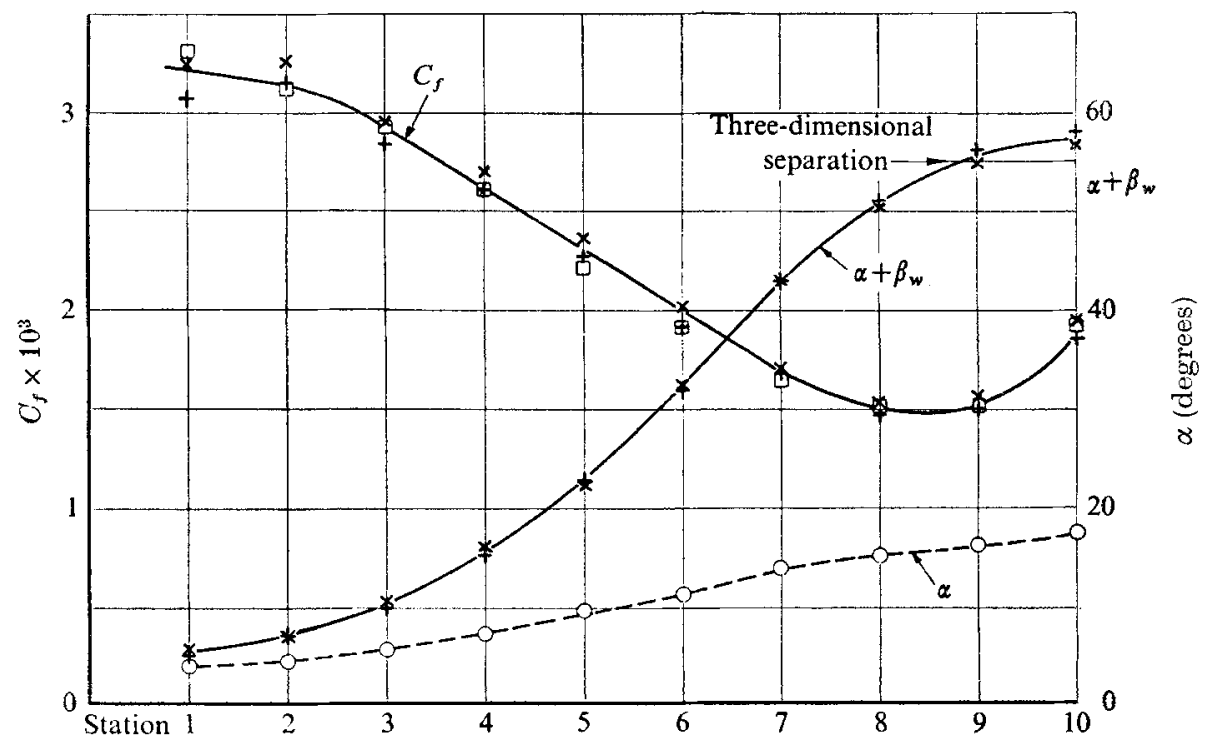

Figure 8. Measured variation of skin-friction coefficient $C_{f}$, flow angle at the boundarylayer edge $\alpha$, and wall shear stress angle $\beta_{w}$. Wall Pitot $(\mathrm{mm}): \times, 0 \cdot 1 ;+, 0 \cdot 2 . \square$, Clauser plots. 
dimensional flow. Investigations of the law of the wall in three-dimensional boundary layers (van den Berg 1975) show that it is probable deviations from the two-dimensional law of the wall will be sufficiently small to neglect for the present purpose. The agreement between the wall Pitot data and the Clauser plot results, which rely on different regions of the law of the wall, give further support for the validity of the methods used to determine the wall shear stress, since equal discrepancies in both regions are unlikely.

Figure 8 also gives the variation with distance from the leading edge of the measured flow angles at the boundary-layer edge $\alpha$ and the measured wall shear angles $\beta_{w}$. The total turning angle $\left(\alpha+\beta_{w}\right)$ is seen to exceed $55^{\circ}$ just in front of station 9 , which means that the wall streamline is parallel to the leading edge there. Several definitions of the separation line in three-dimensional flows have been given in the literature (see e.g. Cooke \& Brebner 1961); but none of them is completely satisfactory. For the infinite swept-wing case, it is clear, however, that the wall streamline parallel to the leading edge must be called the separation line.

It is interesting that the wall shear stress exhibits a minimum near the separation line. In a two-dimensional flow, the absolute value of the wall shear stress is actually also minimum. The fast increase of the wall shear stress behind the separation line which occurs here is somewhat surprising, however.

The variation of some thickness parameters of the three-dimensional boundary layer with distance from the leading edge is given in figure 9 . The plot includes the boundary-layer thickness $\delta_{995}$, where $U / U_{e}=0.995$, the displacement thickness and momentum thickness in the stream direction $\delta_{1}$ and $\theta_{11}$, and the displacement thickness in crosswise direction $\delta_{2}$.

An important indication of the reliability of the boundary-layer data is the accuracy with which the momentum balance between the measuring stations holds. The momentum defect, integrated across the boundary layer, can be calculated from the measured variation of the pressure and wall shear stress, using the momentum integral equations. The momentum defect integral can also be obtained directly from the measured velocity profiles. Figure 10 shows, for the present experiment, the level of agreement of the momentum defect integrals obtained in both ways (i.e. the accuracy with which the momentum balance holds). Comparisons are given for the integrated momentum defects normal and parallel to the leading edge. In both directions, agreement is seen to be good, up to near the separation line.

Tabulated values of the experimental results are given in van den Berg \& Elsenaar (1972), which also includes a polynomial describing the pressure distribution on the flat plate. It is recommended to use this polynomial for boundary-layer calculations, so that differences in the computed results of various calculation methods will be due to the method, not to other approximations to the pressure distribution.

$\dagger$ Later measurements of the wall shear stress direction from oil flow patterns at stations 5 and 7 (van den Berg 1972) suggest that the wall shear stress angles given here may be one or two degrees too small in the neighbourhood of these stations, where the crosswise pressure gradients are large. 


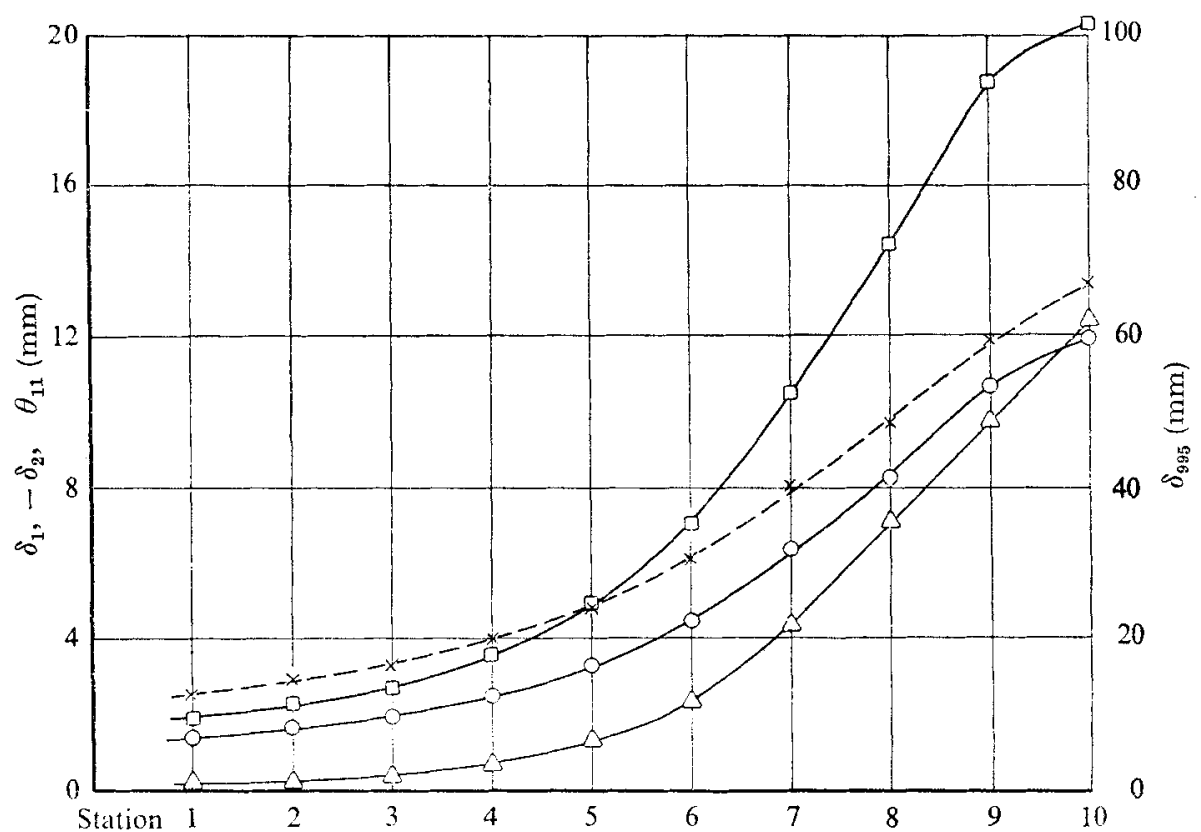

Figune 9. Measured variation of some boundary-layer thickness parameters. $\square, \delta_{1}$; $\triangle, \delta_{2} ; \bigcirc, \theta_{11} ; \times, \delta_{995}$.

$$
\delta_{1}=\int_{0}^{\delta}\left(1-\frac{U_{s}}{U_{e}}\right) d y, \quad-\delta_{2}=\int_{0}^{\delta} \frac{U_{c}}{U_{e}} d y, \quad \theta_{11}=\int_{0}^{\delta}\left(1-\frac{U_{s}}{U_{e}}\right) \frac{U_{s}}{U_{e}} d y
$$

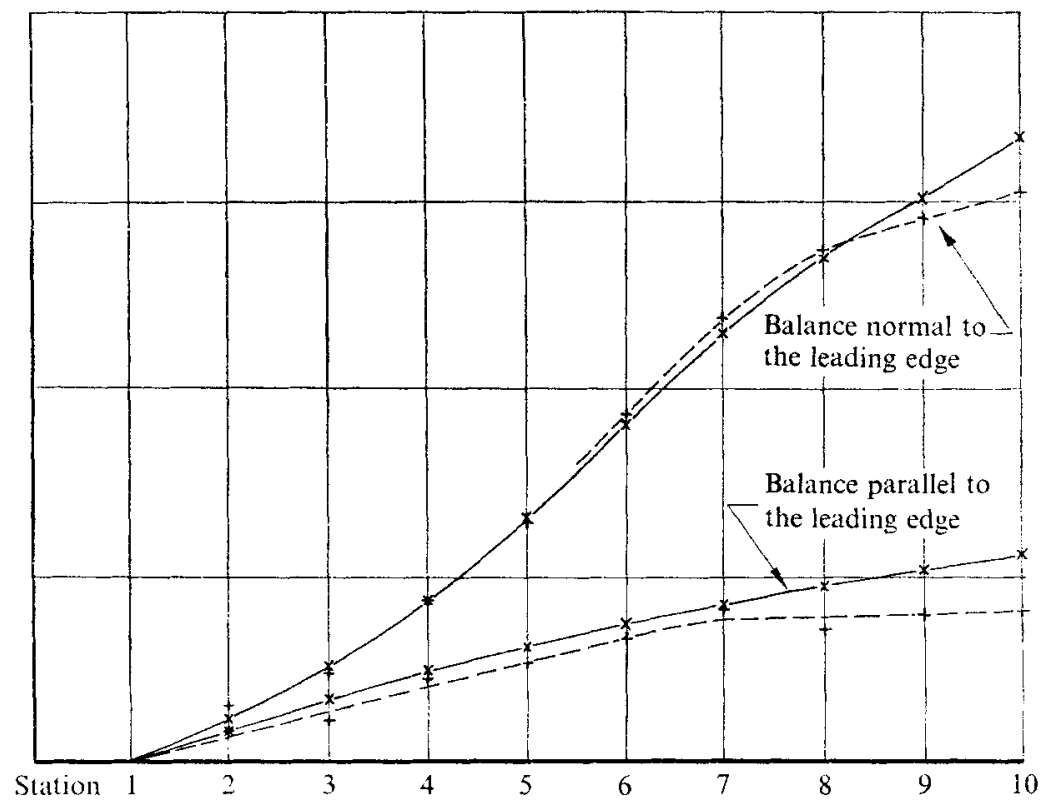

FIGURE 10. Momentum integral check on boundary-layer measurements, assuming infinite swept-wing conditions. Momentum defect integral : - - - , from measured velocity profiles; $\ldots$ - from measured pressure gradient and wall shear stress. 


\section{Method of calculation}

Boundary-layer calculation methods may be divided roughly into two classes: field and momentum integral methods. In the first, the number of independent variables is equal to the number of space dimensions. Momentum integral methods have one independent variable less, owing to an integration of the governing equations over the boundary-layer thickness. The use of integral methods is profitable from a computational point of view. The main drawback is that integral methods require the assumption of velocity profile families. This drawback is particularly severe for three-dimensional boundary layers, because cross-flow families of reasonable accuracy and generality do not (yet) exist. For that reason, a field method has been chosen here. In field methods, assumptions are made about the turbulent shear stress itself. In that case, a generalization from two to three dimensions can be made rather straightforwardly.

The three-dimensional turbulent boundary-layer calculation method employed here will be described briefly. A more detailed exposition has been given by Wesseling \& Lindhout (1971) and Lindhout (1974). The method is closely related to that of Bradshaw (1971); and it is an extension of the two-dimensional method of Bradshaw et al. (1967). This method uses a differential equation for the turbulent shear stress, which takes into account the history of the turbulence. Bradshaw (1971) and Wesseling (1969) have generalized the equation for the turbulent shear stress to three dimensions in different ways, but with the same final result. The transport equations obtained for the turbulent shear stress read, when the $y$ co-ordinate is chosen normal to the wall,

$$
\begin{aligned}
\frac{1}{2 a} \frac{D \tau_{x}}{D t} & =\tau \frac{\partial U_{x}}{\partial y}-\left(\frac{\tau_{\max }}{\rho}\right)^{\frac{1}{2}} \frac{\partial}{\partial y}\left(G \tau_{x}\right)-\left(\frac{\tau}{\rho}\right)^{\frac{1}{2}} \frac{\tau_{x}}{L} \\
\frac{1}{2 a} \frac{D \tau_{z}}{D t} & =\tau \frac{\partial U_{z}}{\partial y}-\left(\frac{\tau_{\max }}{\rho}\right)^{\frac{1}{2}} \frac{\partial}{\partial y}\left(G r_{z}\right)-\left(\frac{\tau}{\rho}\right)^{\frac{1}{2}} \frac{\tau_{z}}{L}
\end{aligned}
$$

Here $U$ and $\tau$ are the resultant velocity and turbulent shear stress, respectively, with components $U_{x}, U_{z}, \tau_{x}$ and $\tau_{z}$. The symbols $a, G$ and $L$ represent empirical functions for which the functions used in the two-dimensional version of the calculation method have been taken (Bradshaw et al. 1967).

The left-hand sides of the equations represent the advection of shear stress. The right-hand-side terms may be called the production, diffusion and dissipation terms, respectively. The production and dissipation terms are dominant in the equation. If the other terms are neglected, the shear stress equation reduces to the well-known mixing-length relation

$$
\tau=L^{2} \rho\left\{\left(\partial U_{x} / \partial y\right)^{2}+\left(\partial U_{z} / \partial y\right)^{2}\right\} \quad \text { with } \quad \tau_{y} / \tau_{z}=\left(\partial U_{x} / \partial y\right) /\left(\partial U_{z} / \partial y\right)
$$

The equations of motion for three-dimensional boundary layers may be written as

$$
\begin{aligned}
& \rho \frac{D U_{x}}{D t}=-\frac{\partial p}{\partial x}+\frac{\partial \tau_{x}}{\partial y}+\mu \frac{\partial^{2} U_{x}}{\partial y^{2}} \\
& \rho \frac{D U_{z}}{D t}=-\frac{\partial p}{\partial z}+\frac{\partial \tau_{z}}{\partial y}+\mu \frac{\partial^{2} U_{z}}{\partial y^{2}}
\end{aligned}
$$




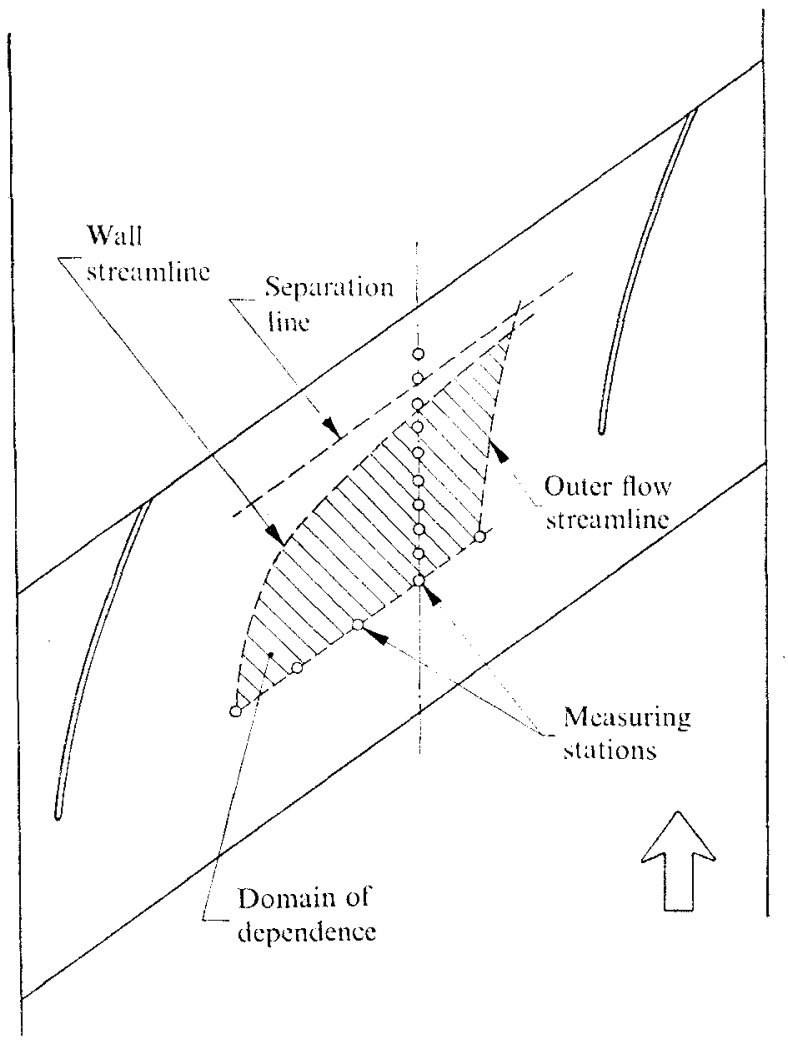

Figure 11. Domain of dependence, deduced from measurements.

Provisionally, the last term in these equations will be neglected (i.e. only the turbulent shear stress will be taken into account). The influence of the viscous forces, which is restricted to a very thin layer close to the surface, will be discussed later.

Together with the continuity equation

$$
\partial U_{x} / \partial x+\partial U_{y} / \partial y+\delta U_{z} / \partial z=0
$$

a system of 5 partial differential equations is obtained. The system of equations is found to be hyperbolic, and to possess 3 characteristics. One characteristic is the normal to the surface. The other two make small angles with the local flow direction; and their projections on the surface coincide with the projection of the local velocity vector. This means that transport of flow properties occurs normal to the surface, and in the local flow direction. This is of interest for the establishment of the domain of influence of the flow at a given point $P$. It is easy to show that the projection of the domain of influence on the surface is the projection of all streamlines in the boundary layer going through the normal to the surface through $P$. This domain is identical to the domain of influence derived by Raetz (1957) for three-dimensional laminar boundary layers.

Figure 11 shows the domain of dependence for the experiment discussed in $\$ 2$. The domain of dependence (i.e. the region in which the flow is determined by the 
measured initial conditions) is bounded by the outer flow streamline through the measuring station at the extreme right and the wall streamline through the station at the extreme left. It should be noted that the wall streamline restricts the calculable region more than will be necessary in practice, since the flow close to the surface is mainly determined by local conditions, which means that the transport of flow properties is of minor importance there.

The five partial differential equations that describe the boundary-layer flow are solved numerically, by means of a simple linear explicit finite-difference scheme, of which the difference molecule is sketched in figure 12. This scheme is a generalization of the two-dimensional Courant-Isaacson-Rees scheme. The solution is assumed to be known at the points $A_{1}$ to $A_{5}$. The flow properties at $B$ are then calculated, using the characteristics through $B$, which intersect the quadrangle $A_{2} A_{3} A_{4} A_{5}$. The difference scheme satisfies the Courant-Friedrics-Levy condition. A thorough discussion of the numerical procedures is given by Lindhout (1974).

The numerical calculations cannot be extended to the surface, since the viscous forces are neglected, and this is not permissible in the viscous sublayer. Close to the surface, however, the variation of the velocity can be described, usually with good accuracy, by the law of the wall. By using the law of the wall, the calculation by finite differences of the thin region adjacent to the wall can be avoided. This has also computational advantages, because the velocity gradients are much larger there than in the remainder of the boundary layer. The use of the simple two-dimensional law of the wall appeared to give incorrect results in three-dimensional flows, however, because the rotation of the velocity vector in the wall law region is neglected. Neither were satisfactory results obtained with some proposed three-dimensional versions of the law of the wall (Nash 1969; Bradshaw 1971; Wesseling \& Lindhout 1971; Nash \& Patel 1971). Finally, a newly-derived law of the wall (van den Berg 1972, 1975) was used with more success. This new wall law describes the variation of both the magnitude and the direction of velocity close to the wall. It takes into account the effect of the external pressure gradient, as well as the inertial effects.

The calculation method described here is not restricted to boundary layers with small cross-flow, or to those under infinite swept-wing conditions (i.e. quasi two-dimensional flows). In fact, there are two versions of the computer program: one for quasi two-dimensional, and one for fully three-dimensional boundary layers. The computation times were found to be acceptable (e.g. $15 \mathrm{~min}$ on a CDC 6600 to calculate the present experiment by the fully three-dimensional program).

\section{Comparison of theory with experiment}

\subsection{External flow}

Before comparing boundary-layer calculations with the results of an experiment, it is sensible to check whether the measured external flow is consistent with the Euler equations for an inviscid flow. In a two-dimensional potential flow, this simply means that the measured dynamic head at the boundary-layer 


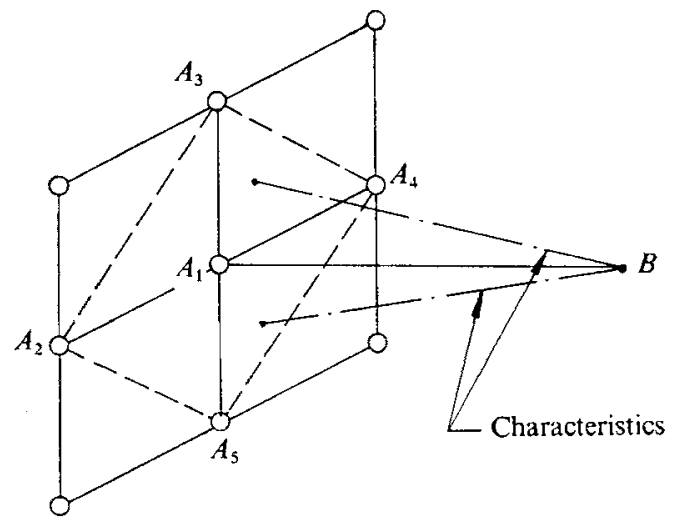

FIGURE 12. Finite-difference molecule, used in the three-dimensional method of calculation.

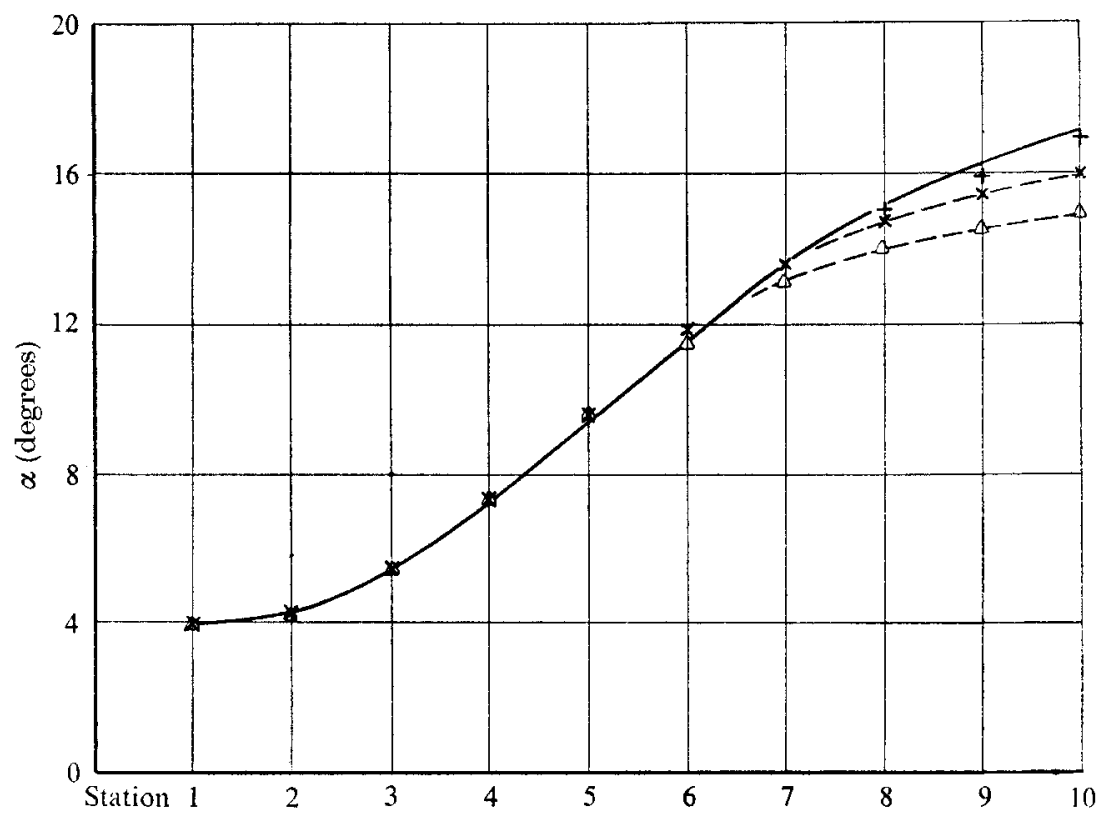

FIGURE 13. Comparison between measured flow angles at the boundary-layer edge and angles derived from pressures measured at the surface, using the Euler equations. - experiment. Calculation: $\Delta$, quasi two-dimensional; $x$, fully three-dimensional; + , the same, corrected for pressure variation across boundary layer.

edge must be equal to the difference between the free-stream total head and the static pressure at the wall, since the static pressure is constant across the boundary layer within the accuracy of the boundary-layer approximation. In threedimensional flows, the static pressure distribution on the wall fixes not only the dynamic head, but also the flow angle at the boundary-layer edge. For infinite swept wings, the relation between the flow angle $\alpha$ and the pressure coefficient $C_{p}$ is simple: $\sin (\Lambda+\alpha) \propto\left(1-C_{p}\right)^{-\frac{1}{2}}$, where $\Lambda$ is the sweep angle. For the general three-dimensional case, the Euler equations must be solved numerically, to compute the external flow field from the pressure distribution on the wall. 
In figure 13 the flow angles $\alpha$ calculated in this way are compared, for the present experiment, with the measured direction of the flow just outside the boundary layer. The quasi two-dimensional calculations of the flow angle are seen to agree very well with the flow-angle measurements over a large distance. Behind measuring station 7 , however, deviations become apparent. These deviations are partly due to an insufficiently accurate approximation to the infinite sweptwing condition far downstream on the test plate. This is demonstrated clearly by the results of the flow angle calculations based on the measured fully threedimensional wall pressure distribution. In the latter case, good agreement is found up to station 8 . The remaining difference between the calculated and measured flow angles is very probably attributable to a small static pressure variation through the boundary layer that seems to exist at the last two stations. Measurements suggest that a static pressure variation of a few per cent of the dynamic head exists at these stations. When a correction is applied for the difference between the pressure at the wall and the boundary-layer edge, good agreement is obtained, up to the last measuring station, as shown in figure 13.

Since three-dimensional separation occurs between station 8 and 9 , it is not to be expected that boundary-layer calculations up to the separation line will be affected by the static pressure variations found at stations 9 and 10 . The external flow angle results suggest that the assumption of a quasi two-dimensional flow may indeed lead to small errors near the separation line. It should be noted, however, that this is not evidenced by the quasi two-dimensional and fully three-dimensional boundary-layer calculations, to be discussed in the next section.

\subsection{Boundary-layer flow}

Three-dimensional turbulent boundary-layer calculations by the method described in $\$ 3$ have been carried out for the present experiment. Apart from the velocity profile at the first measuring station, the shear stress distribution at this station is required as an initial condition for the calculation method employed. The shear stress distribution at station 1 was obtained from the turbulent shear stress equation used in the calculation method, taking into account only the two dominant terms, which means that the equation reduces to the mixinglength relation. The resultant shear stress distribution differs little from that to be expected for a two-dimensional constant-pressure boundary layer, which occurs very nearly at station 1 . Some of the results of the calculations will now be compared with the experimental results.

In figure 14 the calculated and measured displacement thickness $\delta_{1}$ are plotted against distance from the leading edge of the test plate. Various step sizes were applied in the calculations, to check the convergence of the numerical procedure. The truncation error is proportional to the step size, in accordance with the theoretical expectations for the finite-difference scheme employed. The calculated data in the subsequent graphs were obtained at a step size $\Delta y=0.25 \mathrm{~mm}$.

Comparison of the calculated streamwise displacement thickness with experiment shows that good agreement exists up to station 6. Downstream of this station, however, boundary-layer growth is substantially underestimated by the 


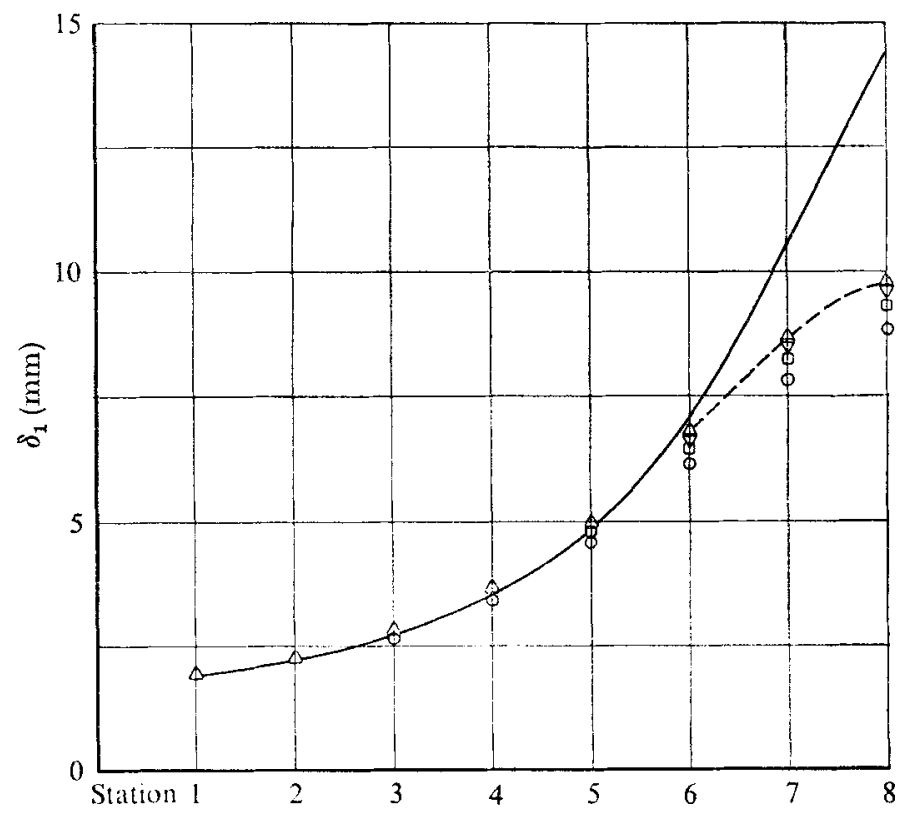

Frgure 14. Comparison of measured with calculated streamwise displacement thickness. Curves also show effect of varying step size in numerical calculations. Quasi two-dimensional calculation; first grid point at $y_{1}=1.0 \mathrm{~mm}$. -

$\begin{array}{lcccc}\text { Step size, } \Delta y(\mathrm{~mm}) & 1.0 & 0.5 & 0.25 & 0.1 \\ & \bigcirc & \square & \nabla & \triangle\end{array}$

theory. The deviations of theory from experiment are even more evident when looking at the wall shear stress direction. Figure 15 gives the measured and calculated wall shear stress angle $\beta_{w}$, relative to the flow direction at the boundary-layer edge. It is clear that, downstream of station 6 , the agreement between theory and experiment becomes very poor. While in the experiment the wall shear stress angle continues to increase (which leads to a three-dimensional separation some distance behind station 8 ), the calculated wall shear stress angle is seen to reach a maximum near station 7 . Further discussion of the discrepancies between theory and experiment at these downstream stations is postponed to the end of this section.

All calculated results discussed up to now were obtained with the quasi twodimensional computer program. These quasi two-dimensional calculations are based on the pressure data in the measurement plane. In figure 15, the results of a quasi two-dimensional and a three-dimensional calculation, using the measured pressure distribution over the whole test plate, are compared. It appears that the differences between the results of the two calculations are negligible. Obviously, the deviations from a really infinite swept-wing pressure distribution are sufficiently small to have no appreciable effect on boundary-layer flow.

Figure 15 also shows the results of a calculation starting with a two-dimensional collateral velocity profile, instead of the slightly skewed velocity profile that was measured at station 1 . It is apparent that the difference in wall shear stress angle 


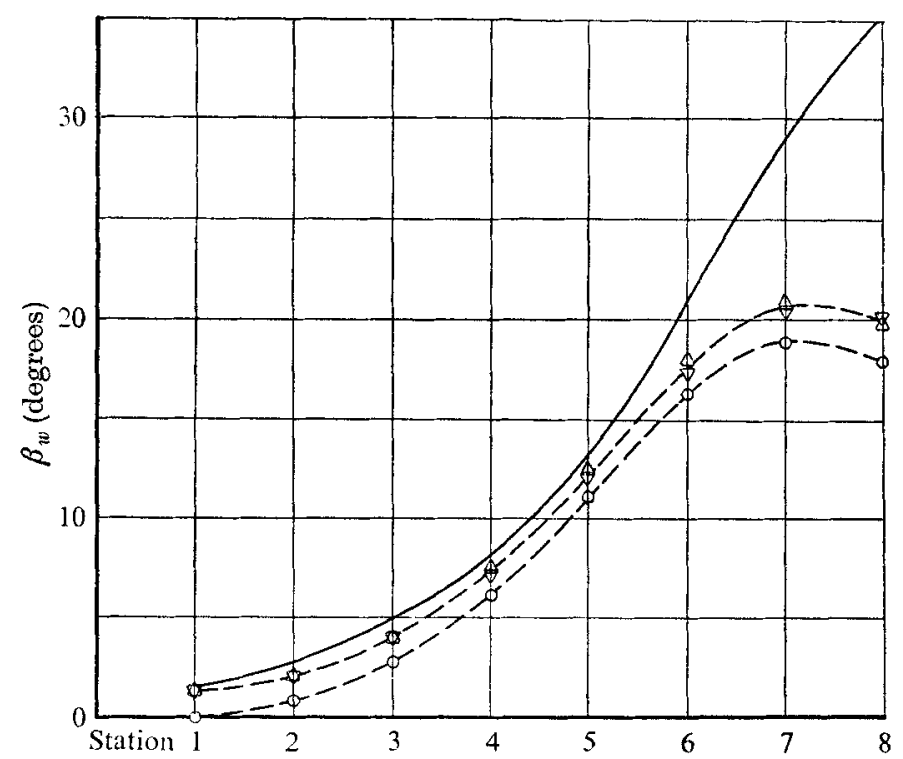

Figure 15. Comparison of measured with calculated wall shear stress angle. Results of a calculation with a collateral initial profile also shown. Step size $\Delta y=0.25 \mathrm{~mm}$; first grid point at $y_{1}=1.0 \mathrm{~mm}$. - - experiment. Calculation: $\nabla$, fully three-dimensional; $\triangle$, quasi two-dimensional; $O$, the same, with $\beta=\beta_{w}=0$ at station 1.

that existed initially neither increases nor decreases during the calculation. This means that a two-dimensional initial profile could be assumed, if this were advantageous from a computational point of view.

As mentioned in $\$ 3$, the numerical calculations are not extended to the surface. Instead, a law of the wall is used as the boundary condition. Figure 16 illustrates the effect of the choice of the law of the wall on the calculated variation of the wall shear stress angle. The results obtained with two wall laws are shown here: the well-known two-dimensional log law,

$$
U / u_{\tau}=(1 / k)\left[\ln \left(y u_{\tau} / v\right)+A\right] \text { with } k=0.41 \text { and } A=2 \cdot 05,
$$

and a new three-dimensional law of the wall, which extends the simple log law by adding correction terms for the pressure gradient and flow acceleration (van den Berg 1975). Figure 16 also shows results for various positions of the first grid point of the numerical scheme. At this grid point the law of the wall is connected with the numerical solution. For a reliable method, the solution should be independent, within certain limits, of the choice of the distance $y_{1}$ of the first grid point from the wall. To check this, the position was varied between $y_{1}=0.5 \mathrm{~mm}$ (which corresponds to a grid point located just outside the viscous sublayer) and $y_{1}=2 \mathrm{~mm}$ (which already constitutes about a sixth of the total boundary-layer thickness for the first stations).

It is evident from figure 16 that the wall shear stress angle is grossly underestimated by the simple two-dimensional law of wall. This must be attributed to the fact that there is substantial rotation of the velocity vector close to the wall, neglected here. It is not surprising that in this case the calculated results depend 


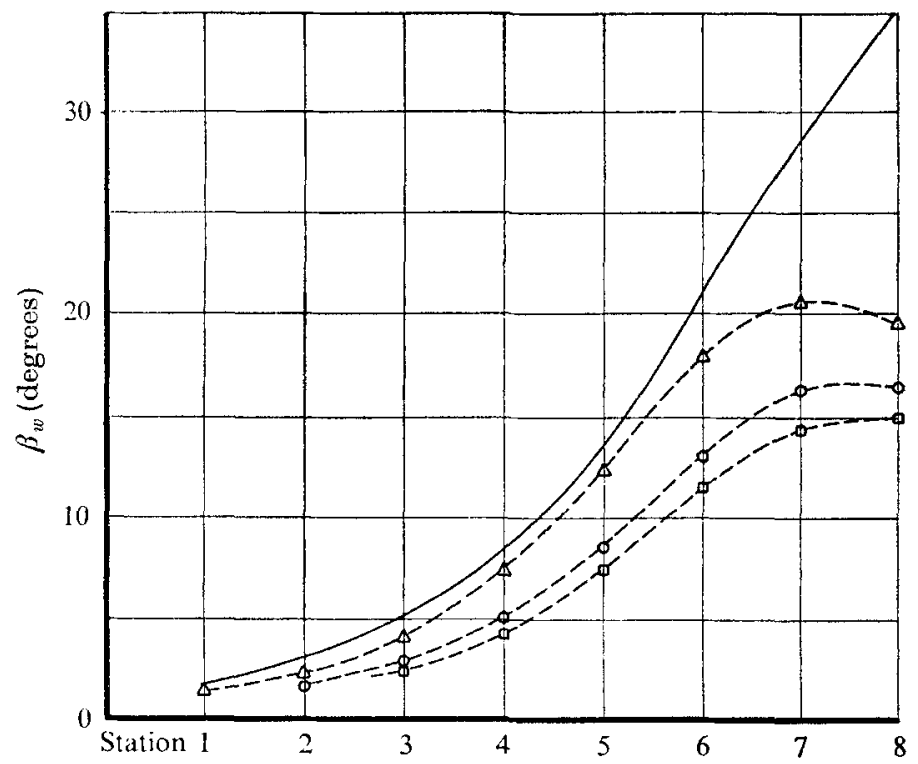

Figure 16. Comparison of measured with calculated wall shear stress angle, for various locations of the first grid point and two laws of the wall. Quasi two-dimensional calculation; step size $\Delta y=0.25 \mathrm{~mm} . y_{1}=1 \mathrm{~mm}$ corresponds to $y u_{\tau} / \nu=60-100$. - , experiment. Calculation, new three-dimensional law of the wall: $\triangle, 0.5<y_{1}<2.0 \mathrm{~mm}$. Calculation, simple two-dimensional law of the wall: $\bigcirc, y_{1}=0.5 \mathrm{~mm}, \square, y_{1}=1.0 \mathrm{~mm}$.

strongly on the choice of the distance $y_{1}$ of the first grid point from the wall. Much better agreement with experiment is seen to exist with the new threedimensional law of the wall. Moreover, results of the calculations appear to be independent of the chosen value for $y_{1}$, within the limits indicated. The new wall law seems to be a good first-order analytical approximation in the wall region of the equations employed for the boundary-layer calculation.

The importance of the use of a good wall law is further illustrated in figure 17, where the measured and calculated skin-friction coefficients $C_{f}$ are compared. When the new wall law is applied, good agreement with experiment is obtained up to station 6 . Further downstream deviations between theory and experiment become apparent, as was the case with the wall shear stress angle and the streamwise displacement thickness. Similar conclusions can be drawn when comparing measured and calculated values of other boundary-layer parameters, such as the streamwise momentum thickness $\theta_{11}$ and the crosswise displacement thickness $\delta_{2}$, which are plotted in figure 18. In figures 5 and 6 , the calculated variation of the magnitude and the direction of the velocity with distance from the wall is indicated by a dashed line for station 7 . Comparison with the measured distributions of the velocity magnitude and angle shows that the general shape of the curves is not much different, but that the calculated velocity defect and velocity rotation are substantially underestimated at this far rearward station.

Though the agreement between theory and experiment is good over quite a distance, the results of the calculations cannot be called satisfactory, in view of the large discrepancies downstream of station 6 . It is particularly important that 


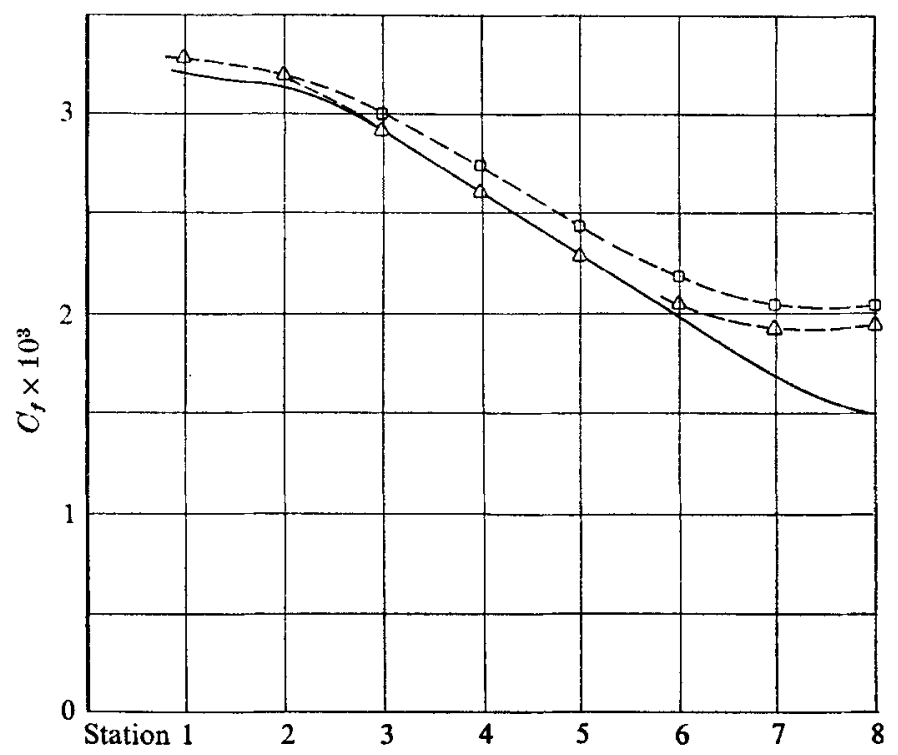

Figure 17. Comparison of measured with calculated skin-friction coefficient, for two laws of the wall. Quasi two-dimensional calculation; step size $\Delta y=0.25 \mathrm{~mm}$; first grid point at $y_{1}=1.0 \mathrm{~mm}$. - experiment. Calculation: $\triangle$, new three-dimensional law of the wall; $\square$, simple two-dimensional law of the wall.

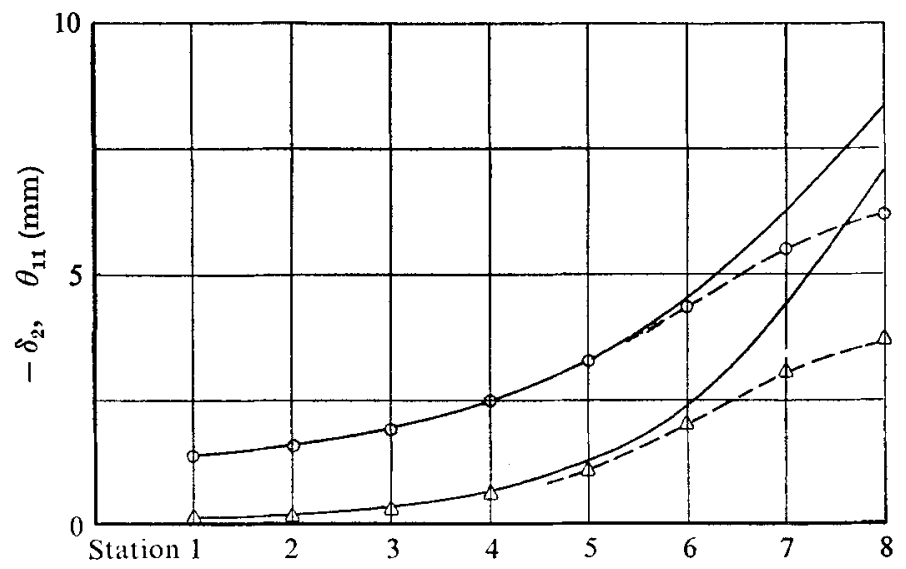

FIGURE 18. Comparison of measured with calculated streamwise momentum thickness and crosswise displacement thickness. Quasi two-dimensional calculation; step size $\Delta y=0.25 \mathrm{~mm}$; first grid point at $y_{1}=1.0 \mathrm{~mm}$. - experiment. Calculation: $\mathcal{O}, \theta_{11}$; $\triangle, \delta_{2}$.

the calculation does not predict separation, while the experimental results indicate that three-dimensional separation occurs not far behind station 8 . When looking at the pressure distribution in figure 4 , it appears that the pressure gradient starts to decrease gradually behind station 5 . It seems that this decrease affects the calculation much more radically than the actual boundary-layer flow reveals. The calculated cross-flow even tends to decrease, as a consequence of the smaller pressure rise, in complete contrast with the measurements. 
In a three-dimensional boundary-layer flow of the type considered here, smaller cross-flows lead to less convergence of the streamlines, and consequently to a smaller growth of the boundary layer. It is not unlikely, therefore, that the discrepancies found may be attributed solely to underestimation of the cross-flow by the theory.

Cross-flows occur owing to the presence of crosswise pressure gradients. At the boundary-layer edge, the crosswise pressure gradient is balanced by the centrifugal force; but, inside the boundary layer, the centrifugal force is too small; so that a cross-flow occurs. The amount of cross-flow is limited by crosswise shear stresses. The apparent underestimation of the cross-flow must be attributed, therefore, to an overestimation of the cross-components of the turbulent shear stresses by the calculation method.

For three-dimensional turbulent boundary-layer calculation methods, based on the assumption of a mixing-length relation or an eddy viscosity (Pierce \& Klinksiek 1971; Krause 1973; Fannelop \& Humphreys 1974), the shear stress direction necessarily coincides with the direction of the velocity gradient, i.e.

$$
\tau_{x} / \tau_{z}=\left(\partial U_{x} / \partial y\right) /\left(\partial U_{z} / \partial y\right)
$$

This is not the case with the present method, because of the presence of advection and diffusion terms in the differential equations employed for the shear stress (see $\S 3$ ). Owing to these terms, the magnitude of the cross-components of the shear stresses appears to diminish. The change in shear stress direction is not at all sufficient, however, to get agreement with experiment.

The fact that essential differences can exist between the direction of shear stress and that of the velocity gradient in three-dimensional turbulent boundary layers is supported by the results of other experiments. East (1973) deduced, from his mean velocity measurements in a three-dimensional boundary layer on a slender wing, the magnitude and direction of shear stress, by applying the equations of motion. He found that, if an eddy viscosity is defined, the eddy viscosity normal to the local mean velocity is only $40 \%$ of that parallel to this velocity. Similar results were obtained by Vermeulen (1971), when analysing his curved duct experiment. Also, the turbulence measurements of Johnston (1970), in a sort of boundary-layer flow induced by a swept-forward facing step, gave shear stress directions that differ substantially from the direction of the velocity gradient. Finally, turbulence measurement carried out recently at NLR in the three-dimensional boundary layer discussed here again indicate that the direction of the shear stress does not, in general, coincide with the direction of the velocity gradient.

It is evident from the foregoing that important alterations in the shear stress equations have to be made, to achieve agreement with experiment up to the separation line. The extension to three dimensions of the shear stress equation that has been used successfully for two-dimensional turbulent boundary layers seems to be less straightforward than has been assumed. This is perhaps after all not very surprising, since the structure of large eddies of the order of the boundary-layer thickness, which play an important role in turbulent shear layers, may very well be affected by the skewing of the velocity profiles in three- 
dimensional boundary layers. In the near future, attempts will be made to improve the turbulent shear stress equations. Turbulence measurements that are being carried out at NLR in the three-dimensional boundary layer described here are expected to contribute towards this.

\section{Conclusions}

A three-dimensional incompressible turbulent boundary-layer experiment has been carried out which is very well suited to verify calculation methods. In this experiment, infinite swept-wing conditions have been approximated with good accuracy. At the first measuring station, the boundary layer was nearly two-dimensional. Three-dimensional separation occurred in the vicinity of the last measuring stations. A thorough analysis of the experimental data indicates that the results are reliable.

The experimental results have been compared with a three-dimensional turbulent boundary-layer calculation method. This uses semi-empirical differential equations for the turbulent shear stress. Good agreement is obtained over a large part of the flow; but increasing deviations becomes apparent as the three-dimensional separation line is approached. These could be attributed to overestimation of the crosswise shear stress in the calculation.

The research discussed here was performed partly under contract with the Royal Netherlands Air Force and the National Agency for Aerospace Programs (NIVR).

\section{REFERENCES}

Berg, B. VAN DEN 1972 NLR Tech. Rep. no. $72111 U$.

BeRg, B. VAN DEN 1975 J. Fluid Mech. 70, 149.

Berg, B. van Den \& ElsenaAr, A. 1972 NLR Tech. Rep. no. 72092 U.

Bradshaw, P. 1971 J. Fluid Mech. 46, 417.

BradHSAW, P. 1973 AGARDograph, no. 169.

Bradshaw, P., Ferriss, D. H. \& Atwell, N. P. 1967 J. Fluid Mech. 28, 593.

Bradshaw, P. \& Terrell, M. G. 1969 NPL Aero Rep. no. 1305.

Clatger, F. H. 1954 J. Aero. Sci. 21, 91.

Cooke, J. C. \& Brebner, G. G. 1961 Boundary Layer and Flow Control (ed. Lachmann). Pergamon.

Cumpsty, N. A. \& Head, M. R. 1970 Aero. Quart. 21, 121.

EAST, L. F. 1973 RAE Tech. Rep. no. 73141.

EAST, L. F. \& Hoxey, R. P. $1969 a$ RAE Tech Rep. no. 69041.

FAST, L. F. \& HoXEY, R. P. $1969 b$ RAE Tech. Rep. no. 69137.

Fannelop, T. K. \& Humphreys, D. A. 1974 A.I.A.A. Paper, no. 74-13.

Francis, G. P. \& Pierce, F. J. 1967 J. Basic Eng. 89, 597.

Hornung, H. G. \& Joubert, P. N. 1963 J. Fluid Mech. 15, 368.

Johnston, J. P. 1960 J. Basic Eng. 82, 233.

Johnston, J. P. 1970 J. Fluid Mech. 42, 823.

KraUSE, E. 1973 AGARD Lecture Series, no. 64.

Lindноut, J. P. F. 1974 NLR Tech. Rep. no. 74159 U. 
148 B. van den Berg, A. Elsenaar, J. P. F. Lindhout and P. Wesseling

Mrchei, R., Cousteix, J. \& Quemand, C. 1972 La Recherche Aérospatiale 1, 1.

NASH, J. F. 1969 J. Fluid Mech. 37, 625.

Nash, J. F. \& PATEL, V. C. 1971 Georgia Inst. Tech. Project Squid Symp.

Pierce, F. J. \& Klinksiek, W. F. 1971 Virginia Polytech. Inst. VPI-E-71-14.

RAETZ, G. S. 1957 Northrop Rep. NAI 58-37.

Rogers, B. K. \& Head, M. R. 1969 Roy. Aero. Soc. Aero. J. 73, 798.

ShaNeBrooK, J. R. \& SUMNER, W. J. 1973 A.I.A.A.J. 11, 950.

SMrth, P. D. 1972 Aero. Res. Council. R. \& M. no. 3739.

Vermedlex, A. 1971 Ph.D. thesis, Department of Engineering, Cambridge University.

Wesseuing, P. 1969 NLR AT 69-01.

Wesseling, P. \& Lindhout, J. P. F. 1971 AGARD Conf. Proc. no. 93. 